Chyle leakage can be caused by abdominal surgery and managed successfully without surgical treatment; however no preventive measures are available. Therefore, we introduce a new method to prevent post-operative chyle leakage. To investigate the role of indocyanine green (ICG) lymphangiography in the reduction of chyle fistula formation after radical resection of right colon cancer.

Five patients with a diagnosis of right colon cancer undergoing laparoscopic radical colectomy with $\mathrm{D} 3$ lymph node dissection were examined in this study. At the end of the operation, two points of $2.5 \mathrm{mg}$ ICG were injected subserosally at the proximal end of the anastomosis ( $1 \mathrm{ml}$ per point). Then the surgical field was screened by using ICG fluorescence to accurately locate the chyle leakage. Chyle leakage was noted and repaired with a Hem-O-Lock. The volume of output of each drain after surgery was measured daily until the patients were discharged.

We were able to observe ICG fluorescence in the lymphatic vessels within 3 minutes of ICG injection. This visualization allowed us to accurately locate and quickly repair chyle leakage within 5 minutes. Clinical observation after surgery and at a 1-month follow-up showed no chyle leakage in all 5 patients.

Indocyanine green lymphangiography can feasibly guide the location and repair of chyle leakage after right colon cancer resection.

Key words: indocyanine green, near infrared fluorescence, right colon cancer, chyle leakage.

Contemp Oncol (Pozn) 2021; 25 (1): 64-67 DOI:https://doi.org/10.5114/wo.2021.105076

\section{Real-time indocyanine green lymphangiography in radical resection of right colon cancer allows the identification of chyle leakage}

\author{
Daqing Cheng ${ }^{1}$, Yunpeng Zhang ${ }^{1}$, Bo Han², Peng Sun ${ }^{1}$, Jue Wang ${ }^{1}$, \\ Quanjun Lin ${ }^{1}$, Ming $\mathrm{Xu}^{1}$
}

\begin{abstract}
${ }^{1}$ Tongren Hospital, Shanghai Jiao Tong University School of Medicine, Shanghai, China ${ }^{2}$ Department of General Surgery, Key Laboratory for Translational Research and Innovative Therapeutics of Gastrointestinal Oncology, Hongqiao International Institute of Medicine, Tongren Hospital, Shanghai Jiao Tong University School of Medicine, Shanghai, China
\end{abstract}

\section{Introduction}

Chyle leakage, usually caused by surgical trauma, may occur after colorectal surgery, with an incidence ranging from 1.0 to $6.6 \%$, depending on the extent of surgery $[1,2]$. The development of chyle leakage can cause the continuous loss of protein and lymphocytes, metabolic depletion, and increased susceptibility to systemic infection [3]. Most patients with chyle leakage can be successfully medically managed without surgical treatment, but for some refractory cases, conservative management fails, and surgical intervention may be required. Unfortunately, reoperative repair of chyle leakage can greatly increase morbidity and mortality [4, 5]. Therefore, an effective strategy is to probe the location of chyle leakage during the operation in real-time and repair the leakage immediately.

Currently, only one near-infrared imaging contrast dye, indocyanine green (ICG), is FDA approved for clinical use in the United States [6-8]. ICG is a safe dye that can be used to visualize bowel microperfusion in real time and has a low incidence of adverse reactions [7]. The advantages of the clinical application of ICG have allowed it to be successfully used in many cancer operations $[9,10]$. For instance, our group and others have used ICG imaging as a highly sensitive method for sentinel lymph node visualization and detection in patients with colon and gastric cancer [11, 12]. The purpose of this study was to evaluate the effectiveness of ICG imaging-guided chyle leakage repair after right colon cancer resection.

\section{Aim}

To investigate the role of ICG lymphangiography in the reduction of chyle fistula formation after radical resection of right colon cancer.

\section{Material and methods}

This is a prospective study. This study was approved by the Tongren Hospital, Shanghai Jiao Tong University School of Medicine Ethics Committee. Informed consent was obtained from all patients undergoing laparoscopic radical colectomy with $\mathrm{D} 3$ lymph node dissection for malignant disease of the right colon from June 2018 to June 2019. 


\section{Patients}

Patients who had received radiotherapy or surgery for colon cancer and patients who were allergic to ICG or iodine and failed follow-up, as well as those with incomplete video data, were excluded from the study. All operations were performed by the same surgeon, with more than 20 years of experience in colon surgery.

\section{Injection of ICG solution and fluorescence imaging}

In the operation, ICG (25 mg per vial, Eisai Pharmaceutical) was prepared by resuspension in $10 \mathrm{ml}$ of sterile water to yield a $2.5 \mathrm{mg} / \mathrm{ml}$ solution. After anastomosis, $1 \mathrm{ml}$ of ICG solution was injected into the subserosa layer of the small intestine at the proximal end of the anastomosis. Near-infrared fluorescence imaging of the surgical site was then performed with a SPY Intraoperative Imaging System (Novadaq Technologies, Mississauga, Ontario, Canada). The presence and location of chyle leakage were noted and recorded. Then, the leakage was repaired with a Hem-O-Lock until no additional leakage was detected.

\section{Post-operative care}

All patients were nursed in the general ward after surgery. The volume of output of each drain was measured and recorded daily until the patients were discharged. Chyle leakage was defined as the presence of non-infectious milky or creamy peritoneal fluid in the drainage tubes, at a volume of $\geq 200 \mathrm{ml} /$ day $[13,14]$. The drained fluid was sent to a laboratory for biochemical testing on postoperative days 1 and 3. Chyle leakage was document- ed and analysed. When the total daily output was less than $200 \mathrm{ml}$, the drains were removed. The patient follow-up period was 1 month after discharge, and the follow-up method is outpatient re-examination or telephone follow-up.

\section{Results}

Visual effect of indocyanine green imagingguided surgical management of chyle leakage

Immediately after injection of the ICG dye solution, visual effects on the surface of the small intestine near the border of the surgical incision were observed, and 3 minutes later the ICG fluorescence was visible in the lymphatic vessels in all 5 patients (Fig. 1A, B). ICG free flow through the lymphatic vessels and the accumulation of ICG fluorescence intensity allowed us to quickly locate chyle leakages in 2 out of 5 patients (Fig. 1C) and successfully repair them with a Hem-O-Lock (Fig. 1D).

\section{Baseline patient characteristics and short-term outcome of this study}

The baseline characteristics of the patients included in this study are summarized in Table 1 . Of the 5 patients included, 2 developed chyle leakage, and the mean time from ICG injection to the first detection of leakage was $3 \pm 0.3$ minutes. The surgical intervention was applied to control the chyle drainage as shown in Fig. 1, and the average time required for lymphatic leak repair guided by ICG imaging was $5 \pm 0.6$ minutes. Patients with chyle leakage repair were discharged within 5 days of clinical routine care, a duration not significantly different from our previously reported length of hospital stay in patients with-
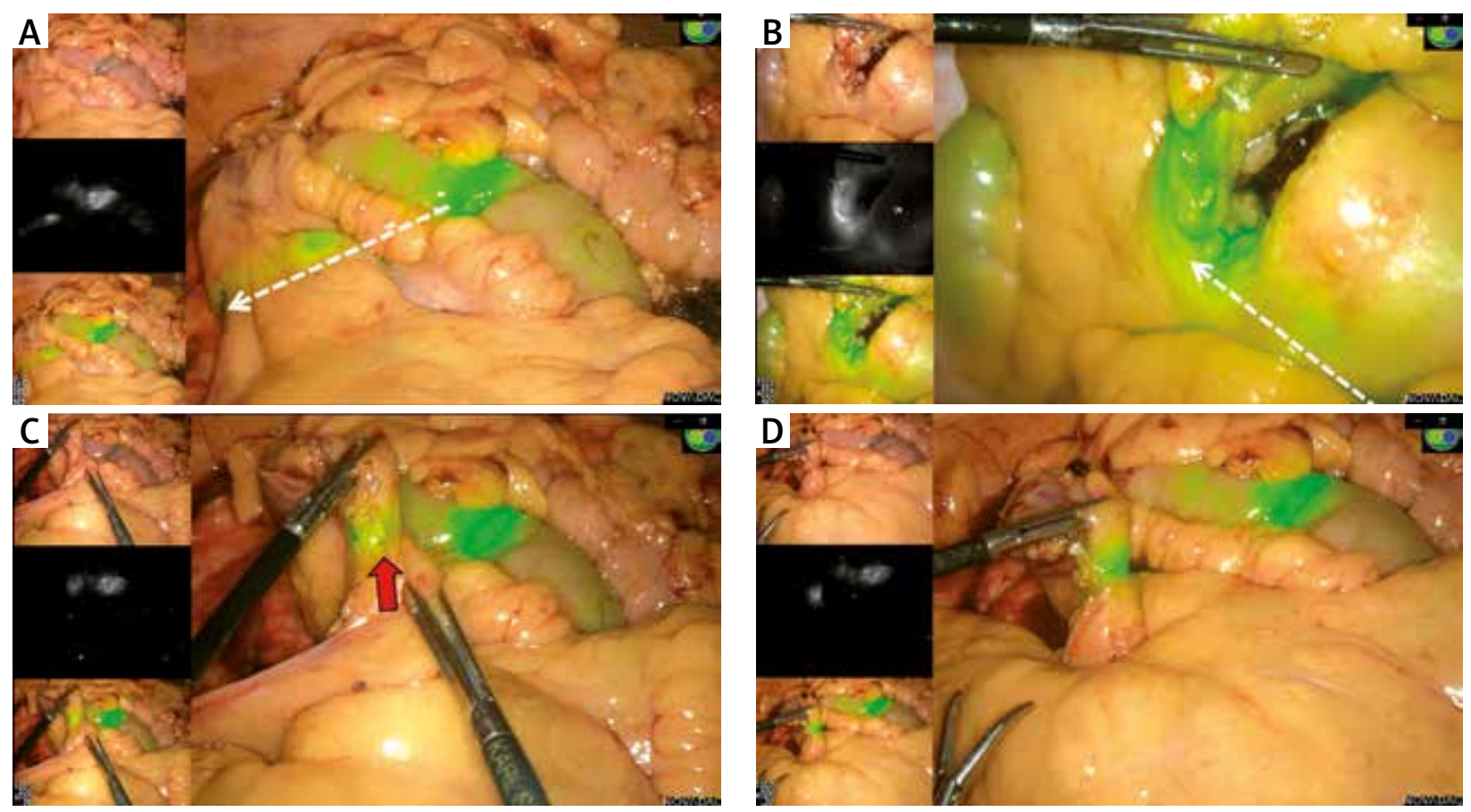

Fig. 1. Visual effect of indocyanine green (ICG) imaging-guided surgical management of chyle leakage. A and B - Free-flowing ICG from the injection sites into the lymphatic vessels in 2 patients (white dotted arrow). C - The ICG fluorescence at the location of the chyle leakage (red arrow). D - The effect of repairing chyle leakage with a Hem-O-Lock 
Table 1. Baseline patient characteristics and short-term outcomes of this study

\begin{tabular}{|c|c|c|c|c|c|c|c|c|}
\hline Patients & Age & Sex & $\mathrm{BMI}$ & $\begin{array}{l}\text { Operation time } \\
\text { (min) }\end{array}$ & ICF & PCF & $\mathrm{NPH}$ & $\begin{array}{c}\text { ICG lymphangiography time } \\
(\min )\end{array}$ \\
\hline 1 & 70 & $M$ & 25.7 & 215 & Yes & No & 6 & 3 \\
\hline 2 & 67 & M & 18.4 & 180 & No & No & 3 & 4 \\
\hline 3 & 44 & $\mathrm{~F}$ & 25.8 & 211 & No & No & 5 & 2 \\
\hline 4 & 72 & M & 27.5 & 200 & Yes & No & 4 & 3 \\
\hline 5 & 64 & $\mathrm{~F}$ & 19.6 & 180 & No & No & 4 & 3 \\
\hline
\end{tabular}

ICF - intraoperative chyle leakage, PCF-postoperative chyle leakage, NPH-number of days of postoperative hospital stay.

out chyle leakage [15]. No complications were found after 1 month of follow-up.

\section{Discussion}

Chyle leakage after surgery is rare, and the incidence varies according to the extent of surgery [2]. The incidence is higher after right colectomy than left colectomy or rectal resection $[16,17]$. One reason for this finding is the presence of abundant lymphatic tributaries close to the cisterna chyle, thus increasing the risk of chyle leakage caused by direct surgical trauma [14]. Chyle leakage can prolong hospital stay, delay the delivery of adjuvant treatment, and even lead to potentially life-threatening conditions if left untreated. All efforts should be made to stop chyle leakage intraoperatively [18], but ligating all vessels during a laparoscopic operation is not realistically feasible. Therefore, early diagnosis and precise repair of chyle leakage during colon surgery would enable effective prevention of post-operative chyle leakage.

Traditional lymphangiography and its derived or improved technologies, such as intranodal lymphangiography and balloon occluded retrograde abdominal lymphatic embolization $[19,20]$, have been used in recalcitrant cases for the diagnosis and treatment of chyle leakage. However, their application is limited because they are invasive and time-consuming, and involve radioactivity. With the development of modern surgery, safe, practical, and convenient chyle leakage assessment methods such as ICG lymphography have been widely used to improve outcomes involving the lymphatic system. Rebecca et al. have shown that ICG lymphangiography facilitates the identification of lymphatic leaks in the groin, and they have optimized their management in challenging cases [21]. Intra-operative ICG lymphangiography has been successfully used to decrease the drainage volume after neck dissection for caners in the head and neck region, as reported by Chan [22]. Both studies demonstrate the advantages of precise identification of the site of the lymphatic leak by direct visualization rather than blind exploration. In our study, we reported the utility of ICG-guided fluorescence imaging for localizing the site of lymphatic leakage and performing repair during lymph node dissection in patients with right colon cancer. ICG was excreted into the lymphatic channels within 3 minutes in all 5 patients and indicated the continuous leakage of lymphatic fluid with the accumulation of fluorescence intensity of ICG in 2 patients during the operation. The real-time ICG imaging allowed us to quickly and clearly identify and repair the intraoperative chyle leakage (ICF). Our results demonstrated that ICG-guided lymphatic leakage markedly decreased the volume of the drainage in those 2 patients. Therefore, this procedure is an effective approach for the precise location and repair of ICF as well as for preventing post-operative chyle leakage formation, as confirmed during a 1-month follow-up without chyle leakage recurrence.

To our knowledge, this is the first application of ICG lymphography to prevent chyle leakage after resection of right colon cancer. But the current study has some limitations. The number of patients enrolled was small, and the study was restricted to patients with right colon cancer, thus potentially affecting the evaluation of the application potential of ICG lymphangiography in preventing postoperative chyle leakage formation. Despite these limitations, the pattern of chyle leakage control in this study provides helpful insights into the management of chyle leakage after resection of right colon cancer.

\section{Conclusions}

In conclusion, ICG lymphangiography can be used to locate and guide the repair of chyle leakage after resection of right colon cancer, thereby preventing the formation of postoperative chyle leakage.

\section{Acknowledgments}

The study was funded by the National Natural Science Foundation of China (81702333 and 81401958).

The authors declare no conflict of interest.

\section{References}

1. Lee SY, Kim CH, Kim YJ, Kim HR. Chylous ascites after colorectal cancer surgery: risk factors and impact on short-term and longterm outcomes. Langenbecks Arch Surg 2016; 401: 1171-1177.

2. Weniger M, D'Haese JG, Angele MK, Kleespies A, Werner J, Hartwig W. Treatment options for chylous ascites after major abdominal surgery: a systematic review. Am J Surg 2016; 211: 206-213.

3. Steven BR, Carey S. Nutritional management in patients with chyle leakage: a systematic review. Eur J Clin Nutr 2015; 69: 776-780.

4. Ramos W, Faintuch J. Nutritional management of thoracic duct fistulas. A comparative study of parenteral versus enteral nutrition. JPEN J Parenter Enteral Nutr 1986; 10: 519-521. 
5. Alexiou C, Watson M, Beggs D, Salama FD, Morgan WE. Chylothorax following oesophagogastrectomy for malignant disease. Eur J Cardiothorac Surg 1998; 14: 460-466.

6. Pei G, Liu Y, Liu Q, et al. The safety and feasibility of intraoperative near-infrared fluorescence imaging with indocyanine green in thoracoscopic sympathectomy for primary palmar hyperhidrosis. Thorac Cancer 2020; 11: 943-949.

7. Jiang JX, Keating JJ, Jesus EM, et al. Optimization of the enhanced permeability and retention effect for near-infrared imaging of solid tumors with indocyanine green. Am J Nucl Med Mol Imaging 2015; 5: 390-400.

8. Garski TR, Staller BJ, Hepner G, Banka VS, Finney RA Jr. Adverse reactions after administration of indocyanine green. JAMA 1978; 240: 635.

9. Reuthebuch O, Häussler A, Genoni M, et al. Novadaq SPY: intraoperative quality assessment in off-pump coronary artery bypass grafting. Chest 2004; 125: 418-424.

10. Spartalis E, Ntokos G, Georgiou K, et al. Intraoperative indocyanine green (ICG) angiography for the identification of the parathyroid glands: current evidence and future perspectives. In Vivo 2020; 34: 23-32.

11. Kim DW, Jeong B, Shin IH, et al. Sentinel node navigation surgery using near-infrared indocyanine green fluorescence in early gastric cancer. Surg Endosc 2019; 33: 1235-1243.

12. Ankersmit M, Bonjer HJ, Hannink G, Schoonmade LJ, van der Pas M, Meijerink W. Near-infrared fluorescence imaging for sentinel lymph node identification in colon cancer: a prospective single-center study and systematic review with meta-analysis. Tech Coloproctol 2019; 23: 1113-1126.

13. Kaas R, Rustman LD, Zoetmulder FA. Chylous ascites after oncological abdominal surgery: incidence and treatment. Eur J Surg Oncol 2001; 27: 187-189.

14. Baek SJ, Kim SH, Kwak JM, Kim J. Incidence and risk factors of chylous ascites after colorectal cancer surgery. Am J Surg 2013; 206: 555-559.

15. Zhang Y, Xin Y, Sun P, et al. Factors associated with failure of Enhanced Recovery After Surgery (ERAS) in colorectal and gastric surgery. Scand I Gastroenterol 2019; 54: 1124-1131.

16. Nishigori H, Ito M, Nishizawa Y, et al. Postoperative chylous ascites after colorectal cancer surgery. Surg Today 2012; 42: 724-728.

17. Lu XR, Lin HM, Chi P. [Treatment of chyle leak following radical resection for colorectal cancer]. Zhonghua Wei Chang Wai Ke Za Zhi 2010; 13: 808-810.

18. Crumley RL, Smith JD. Postoperative chylous fistula prevention and management. Laryngoscope 1976; 86: 804-813.

19. Chick JF, VanBelkum A, Yu V, et al. Balloon-occluded retrograde abdominal lymphangiography and embolization for opacification and treatment of abdominal chylous leakage. J Vasc Interv Radiol 2017; 28: 616-618.

20. Kawasaki R, Sugimoto K, Fujii M, et al. Therapeutic effectiveness of diagnostic lymphangiography for refractory postoperative chylothorax and chylous ascites: correlation with radiologic findings and preceding medical treatment. AJR Am J Roentgenol 2013; 201: 659-666.

21. Rebecca AM, Mahabir RC, Pflibsen L, Hillberg N, Jensen C, Casey WJ. Indocyanine green lymphangiography as an adjunct for the optimal identification and management of lymphatic leaks in the groin. J Reconstr Microsurg 2019; 35: 83-89.

22. Chan J, Wong S, Wei WI. Real time indocyanin green near infrared lymphangiography for the reduction of drainage volume after neck dissection. Oral Oncol 2018; 78: 52-55.

\section{Address for correspondence}

\section{Bo Han}

Department of General Surgery

Key Laboratory for Translational Research

and Innovative Therapeutics of Gastrointestinal Oncology

Hongqiao International Institute of Medicine

Tongren Hospital

Shanghai Jiao Tong University School of Medicine

1111 XianXia Road, Shanghai 200336, China

e-mail: hb3616@shtrhospital.com

Submitted: 11.06 .2020

Accepted: 22.08 .2020 